\title{
AN APPROACH TO EXTENDING THE RELATIONAL DATABASE MODEL FOR HANDLING INCOMPLETE INFORMATION AND DATA DEPENDENCIES
}

\author{
HO THUAN, HO CAM HA
}

\begin{abstract}
In this paper we propose a new approach to extending the relational database model. This approach is based on the concept of similarity based fuzzy relational database and somewhat of new viewpoint on redundancy. It is shown that, in such an extended database model, we can capture imprecise, uncertain information. The formal definition of fuzzy functional and multivalued dependencies in this study allows a sound and complete set of inference rules. This paper describes an ongoing work. We state some open problems to be solved in order to render our approach more operational.
\end{abstract}

Tóm tăt. Bài báo đề xuất một cách tiếp cận mới để mơ rộng mô hình cơ sở dữ liệu quan hệ. Cách tiếp cận này dựa trên khái niệm cơ sở dũ̃ liệu mờ tương tự và một quan điểm mới về dư thừa dũ liệu. Với mô hình cơ sở dũ liệu như vậy có thể nắm bắt được những thông tin không chính xác, không chắc chắn. Định nghĩa về phụ thuộc hàm mờ và phụ thuộc đa trị mờ trong bài báo cho một tập các luật suy dẫn xác đáng và đầy đư.

\section{INTRODUCTION}

Database systems have been extensively studied since Codd [3] proposed the relational data model. Such database systems do not accept uncertain and imprecise data. In fact, the value of an object's attribute may be completely unknown, incompletely known (i.e., only a subset of possible values of the attribute is known), or uncertain (e.g. a probability or possibility distribution for its value is known). In addition, the attribute may not be applicable to some of the objects being considered and, in certain cases, we may not known whether the value even exists, or not. Many approaches to that problem have been proposed. One of them is "A fuzzy representation of data for relational database" [2], which is suggested by P. Buckles and E. Petry. In [2] a structure for representing inexact information in the form of a relational database is presented. The structure differs from ordinary relational database in two important respects: value of an attribute of an object need not be single value and a similarity relation is required for each domain set of the database. In a fuzzy database proposed by these authors, a tuple is redundant if it can be merged with another through the set union of corresponding domain values. The merging of tuple, however, is subject to constraints on some similar thresholds. Within this conception, in a fuzzy relation with no redundant tuples and each domain similarity relation formulated according to $\mathrm{T} 1$ transitivity, each tuple represents information of an object, and each value of an attribute (called domain value) consists of one or more elements from the domain base set. At this point, there is an emphatic notice that elements of each domain value must be similar enough to each other (i.e. similarity degree of every couple of elements is not less than the given threshold).

The work reported here is quite distinct from that of P. Buckles and E. Petry in that the elements of each domain value are not required to be similar enough according to the threshold. This idea allows each domain value to contain elements, which even are not very similar and represent the possibilities that can be happened. Therefore, to model a relational database by using this approach will preserve not only the exact information but also the nuances of fuzzy uncertainty.

This paper is organized as follows. Notations and basic definitions related to fuzzy relational data model and similarity relation, are reviewed in Section 2 to get an identical understanding of terminology. A new definition about tuple redundant is presented in Section 3. Section 4 contains 
definition of functional dependency in this scene. The soundness and completeness of the set of axioms, which is similar with Amstrong's axioms in the traditional relational database, will be proved in this section. In Section 5, we propose a formal definition of fuzzy multivalued dependency and the inference rules.

\section{BACKGROUND}

First, similarity relations are described as defined by Zadeh [9]. Then the basic concepts of fuzzy relational database model are reviewed.

Similarity relations are useful for describing how similar two elements from the same domain are.

Definition 2.1. ([5]) A similarity relation, $s_{D}(x, y)$, for a given domain $D$, is a mapping of every pair of elements in the domain onto the unit interval $[0,1]$ with the three following properties, $\forall x, y, z \in D$ :

1. Reflexivity

$S_{D}(x, x)=1$

2. Symmetry

$S_{D}(x, y)=S_{D}(y, x)$

3. Transitivity

$S_{D}(x, z) \geq \operatorname{Max}_{y}\left(\operatorname{Min}\left[S_{D}(x, y), S_{D}(y, z)\right]\right)$

or 3 '. Transitivity

where $*$ is arithmetic multiplication)

For each domain $j$ in a relational database, a domain base set $D_{j}$ is understood. Domains for fuzzy relational databases will be either discrete scalars or discrete numbers drawn from either a finite or infinite set. A domain value $d_{i j}$, where $i$ is the tuple index, is defined to be a subset (not empty) of its domain base set $D_{j}$. Let $2^{D_{j}}$ denote a set of any non-null member of the powerset of $D_{j}$.

Definition 2.2. ([2]) A fuzzy relation, $r$, is a subset of the set cross product $2^{D_{1}} \times \cdots \times 2^{D_{m}}$.

Definition 2.3. ([2]) A fuzzy relation tuple, $t$, is any member of $2^{D_{1}} \times \cdots \times 2^{D_{m}}$.

An arbitrary tuple is of the form $t_{i} \in r, t_{i}=\left(d_{i 1}, d_{i 2}, \ldots, d_{i m}\right), d_{i j} \subseteq D_{j}$. For example:

\begin{tabular}{|c|c|c|}
\hline Name & Car_color & Job \\
\hline JJohn $\}$ & \{green, blue, pink $\}$ & \{doctor, physician, dentist, farmer $\}$ \\
\hline
\end{tabular}

\section{REDUNDANCY AND DETERMINANCY PROPERTIES}

In a nonfuzzy database, a tuple is redundant if it is exactly the same as another tuple. In fuzzy database of P.Buckles and E.Petry [2], a tuple is redundant if it can be merged with another without violating

$\operatorname{LEVEL}\left(D_{j}\right)=\operatorname{THRES}\left(D_{j}\right), j=1,2, \ldots, m$, where

$\operatorname{THRES}\left(D_{j}\right)=\min _{i}\left\{\min _{x, y \in d_{i j}}[s(x, y)]\right\} \quad[2]$

In a given domain $D_{j}, x, y \in D_{j}$, if $s(x, y) \geq \operatorname{LEVEL}\left(D_{j}\right)$ then we write down $x \sim y$. Obviously, $\sim$ is a binary relation on $D_{j}$.

Lemma 3.1. $\sim$ is an equivalence relation.

Proof. $\forall x \in D_{j}, s(x, x)=1$, so $s(x, x) \geq \operatorname{LEVEL}\left(D_{j}\right)$, we have $x \sim x$.

Symmetry property of $\sim$ relation is easily implied from the symmetry property of a similarity measure. $\forall x, y, z \in D_{j}$, if $s(x, y) \geq \operatorname{LEVEL}\left(D_{j}\right)$ and $s(y, z) \geq \operatorname{LEVEL}\left(D_{j}\right)$, from (T1) transitivity we have $s(x, z) \geq \operatorname{LEVEL}\left(D_{j}\right)$.

Thus, $\sim$ is an equivalence relation and induces a unique partition in $D_{j}$.

In a fuzzy relational scheme suggested by Buckles and Petry [2], each domain value may consist of many elements, all of which belong to the same equivalence class partitioned by the $\sim$ relation. 
According to these authors, two tuples are redundant to each other if on every attribute, the domain value of each tuple includes representatives of the same equivalence class. To a certain meaning, if we consider an equivalence class (of the $\sim$ relation) as a branch of possibilities that may happen, the model of P. Buckles and E. Petry will allow only to capture information of the objects, of which the known information about each attribute belongs to only one branch of possibilities. The branch of possibilities mentioned here is considered to be shown by values, which are, although not equal to each other, but closed enough to each other according to the measure of a similarity relation. However, in fact there can be uncertain information about an object, on an attribute of that there are many possibilities which are far different to each other. In the above example, John may be a doctor, a physician, a dentist (or any position in medical profession), but John may be also a farmer. John has a green car, or a pink one, but he may have two cars, one is blue and the other is pink. And it is not excluded that John has all the three cars which are green, blue and pink. If a group of possibility branches is considered necessary to keep as it identifies a full information in this case, the model in [2] should be expanded, and we have tried to do this. Suppose that with each Dj there is a $\operatorname{LEVEL}(\mathrm{Dj})$ for an identified similarity on this domain, two tuples are said to be redundant to each other if they have the same group of possibilities on each attribute.

Definition 3.1. In fuzzy relation $r$, two tuples $t_{i}=\left(d_{i 1}, d_{i 2}, \ldots, d_{i m}\right)$ and $t_{k}=\left(d_{k 1}, d_{k 2}, \ldots, d_{k m}\right)$, $i \neq k$ are redundant if

$\forall x \in d_{i j} \exists x^{\prime} \in d_{k j}: x \sim x^{\prime}, \forall j=1,2, \ldots, m$ and vice versa, i.e.

$\forall x \in d_{k j} \exists x^{\prime} \in d_{i j}: x \sim x^{\prime}, \forall j=1,2, \ldots, m$.

As $t_{i}$ and $t_{k}$ are equitable in the above definition, the notation $t_{i} \approx t_{k}$ is used to denote that $t_{i}$ and $t_{k}$ are redundant.

Lemma 3.2. $\approx$ is an equivalence relation on the fuzzy relation $r$.

Proof. It is clear that, for every tuple $t_{i}$ of $r, t_{i} \approx t_{i}$ from reflexivity of $\sim$ relation.

Obviously, if $t_{i} \approx t_{k}$ then $t_{k} \approx t_{i}$.

Suppose that $t_{i} \approx t_{k}$ and $t_{k} \approx t_{h}$. Consider arbitrary domain $D_{j}$, if $x \in d_{i j}$ then $\exists x^{\prime} \in d_{k j}: x \sim x^{\prime}$ (from $t_{i} \approx t_{k}$ ). Since $x^{\prime} \in d_{k j}$, we have $\exists x^{\prime \prime} \in d_{h j}: x^{\prime} \sim x^{\prime \prime}$ (from $t_{k} \approx t_{h}$ ). We also have $x \sim x^{\prime \prime}$ by transitivity of $\sim$ relation. Similarly, if $x \in d_{h j}$ we have $\exists x^{\prime \prime} \in d_{i j}: x \sim x^{\prime \prime}$.

Thus, redundant $(\approx)$ is an equivalence relation on $\mathrm{R}$ and induces a unique partition in $r$.

An example of a fuzzy relation with similarity relations:

\begin{tabular}{|l|l|l|l|}
\cline { 2 - 4 }$t_{1}$ & Name & Car_color & Job \\
\cline { 2 - 4 }$t_{2}$ & John & green, blue, pink & actor, teacher \\
$t_{3}$ & Johan & black, magent & aconductor, instructor \\
$t_{4}$ & Melia & white, pink & artist \\
$t_{5}$ & Tom & black, light_milk & artist \\
\cline { 2 - 3 } & & Tilot \\
\hline
\end{tabular}

Fig. 1. A fuzzy relation

If it is assumed that $\operatorname{LEV}(\mathrm{Name})=0.6$ then $\sim$ relation partitions Dom (Name) by three equivalence classes:

\{John, Johan $\} ;\{$ Elina, Melina $\} ;\{$ Tom $\}$

It is also assumed that $\operatorname{LEV}\left(\right.$ Car_color$_{\text {) }}$ and $\operatorname{LEV}(\mathrm{Job})$ are given such that

Dom(Car_color) and Dom(Job) are partitioned as follow

$\{\{$ green, blue, black $\},\{$ pink, magenta, red $\},\{$ white, light_milk\}\}

$\{\{$ actor, conductor, artist $\},\{$ teacher, instructor $\},\{$ pilot $\}\}$ 
Thus in $r 1$ above, $t_{1}$ is redundant for $t_{2}$ and $t_{3}$ is redundant for $t_{4}$.

\begin{tabular}{|l|l|l|l|l|l|}
\hline & John & Johan & Elina & Melina & Tom \\
\hline John & 1.0 & 0.6 & 0.0 & 0.0 & 0.0 \\
\hline Johan & 0.6 & 1.0 & 0.0 & 0.0 & 0.0 \\
\hline Elina & 0.0 & 0.0 & 1.0 & 0.8 & 0.0 \\
\hline Melina & 0.0 & 0.0 & 0.8 & 1.0 & 0.0 \\
\hline Tom & 0.0 & 0.0 & 0.0 & 0.0 & 1.0 \\
\hline
\end{tabular}

Fig. 2. Similarity relation for Dom(Name)

\section{FUZZY FUNCTIONAL DEPENDENCY AND A SET OF SOUND AND COMPLETE INFERENCE RULES}

Let $r$ is a fuzzy relation with $\mathrm{m}$ attributes, these according to $\mathrm{m}$ domains $D_{1}, D_{2}, \ldots, D_{m}$, we said that $r$ is an instance of $R$, which is called a relation scheme on $U, U=\left\{A_{1}, A_{2}, \ldots, A_{m}\right\}$. Suppose that $X$ is a set of attributes $(X \subseteq U)$; two tuples $t_{1}, t_{2} \in r, t_{1}=\left(d_{11}, d_{12}, \ldots, d_{1 m}\right)$ and $t_{2}=\left(d_{21}, d_{22}, \ldots, d_{2 m}\right)$, we said $t_{1}, t_{2}$ are redundant each other on $X$ and write

$t_{1}[X] \approx t_{2}[X]$ if $\forall x \in d_{1 j} \exists x^{\prime} \in d_{2 j}: x \sim x^{\prime}$, and vice versa, i.e.

$\forall x \in d_{2 j} \exists x^{\prime} \in d_{1 j}: x \sim x^{\prime}, \forall j: A_{j} \in X$.

Definition 4.1. A fuzzy functional dependency $X \approx Y$ is said to be hold in a fuzzy relation $r$ if for every pairs of tuple $t_{1}, t_{2} \in r$ :

$$
t_{1}[X] \approx t_{2}[X] \text { implies that } t_{1}[Y] \approx t_{2}[Y] .
$$

In what follows we assume that we are given a fuzzy relational schema with set of attribute $U$, the universal set of attributes, and a set of fuzzy functional dependencies $F$ involving only attributes in $U$. The inference rules, which similar with Amstrong's axioms are:

FFD1: Reflexivity If $Y \subseteq X$ then $X \approx Y$

FFD2: Augmentation If $X \approx Y$ holds, then $X Z \approx Y Z$ holds

FFD3: Transitivity If $X \approx Y$ and $Y \approx Z$ hold, then $X \approx Z$ holds

Lemma 4.1. The set of FFD axioms (FFD1-FFD3) are sound. That is, if $X \approx Y$ is deduced from $F$ using the axioms, then $X \approx Y$ is true in any relation in which the dependencies of $F$ are true.

Proof.

(FFD1)

The reflexivity axiom is clear sound.

(FFD2)

Suppose $t_{1}, t 2 \in r$ such that $t_{1}[X Z] \approx t_{2}[X Z]$

then by definition of " $\approx$ " we have $t_{1}[X] \approx t_{2}[X]$.

From $X \approx Y$ we have $t_{1}[Y] \approx t_{2}[Y]$

(1) means $\forall x \in d_{1 j} \exists x^{\prime} \in d_{2 j}: x \sim x^{\prime}$, and vice versa $\forall j: D_{j} \in X Z$.

(2) means $\forall x \in d_{1 j} \exists x^{\prime} \in d_{2 j}: x \sim x^{\prime}$, and vice versa $\forall j: D_{j} \in Y$.

So we have

$$
\forall x \in d_{1 j} \exists x^{\prime} \in d_{2 j}: x \sim x^{\prime}, \text { and vice versa } \forall j: D_{j} \in Y Z .
$$

It means $X Z \approx Y Z$.

$$
\text { If } \begin{aligned}
t_{1}[X] \approx t_{2}[X] \text { then we have } t_{1}[Y] \approx t_{2}[Y] \text { from } X \approx Y \\
\text { and } t_{1}[Z] \approx t_{2}[Z] \text { from } Y \approx Z .
\end{aligned}
$$

The following inference axioms are infered from the above axioms 
FFD4 : Union

If $X \approx Y$ and $X \approx Z$ hold, then $X \approx Y Z$ holds.

FFD5 : Decomposition

If $X \approx Y Z$ holds, then $X \approx Y$ and $X \approx Z$ hold.

FFD6 : Pseudotransitivity

Procedure of proof for the completeness of above inference axioms is very similar to the classical case.

Theorem 4.1. The set of axioms (FFD1-FFD2) are sound and complete.

\section{FUZZY MULTIVALUED DEPENDENCY AND SET OF INFERENCE RULES}

In the fuzzy paradigm, let $R$ be a relation scheme and let $X$ and $Y$ be subsets of $R$. In a relation $r$, an instance of $R$, for $X$-value $x$ we define

$X_{r}(x)=\left\{x^{\prime} \mid \exists t \in r\right.$, such that $\left.t[X]=x^{\prime}, x \approx x^{\prime}\right\}$.

$Y_{r}(x)=\left\{y \mid \exists t \in r\right.$, such that $\left.t[X] \in X_{r}(x), t[Y]=y\right\}$.

Let $Z=R-X Y$. It is clear that $Y_{r}(x)$ is independent of $Z$-values. We say that $Y_{r}(x)$ is equivalent to $Y_{r}(x z)$ if for every y of one, there is existing $y^{\prime}$ of the other such that $y \approx y^{\prime}$ and vice versa. The fuzzy equivalence of two set $Y$-value $\left(Y_{r}(x)\right.$ and $\left.Y_{r}(x z)\right)$ can be reperesented as $Y_{r}(x) \cong Y_{r}(x z)$.

Definition 5.1. A fuzzy multivalued dependency (FMVD) $m$ on a scheme $R$, is a statement $m$ : $X \approx \longrightarrow$, where $X, Y$ are subsets of $R$. Let $Z=R-X Y$. A relation $r$ on the scheme $R$ obeys the FMVD $m: X \approx \longrightarrow Y$ if for every $X Z$-value $x z$ that appears in $r$ we have $Y_{r}(x) \cong Y_{r}(x z)$.

Example:

$\begin{array}{ccc}r 2 & Y \text { (Courses) } & Z \text { (Student) } \\ a, b, c & g, h & z 1 \\ a^{\prime}, c^{\prime} & g^{\prime}, i & z 2 \\ a, c^{\prime} & g, i^{\prime} & z 1^{\prime} \\ a^{\prime}, c & g^{\prime}, h^{\prime} & z 2^{\prime}\end{array}$

Fig. 3. A fuzzy relation

$$
\begin{array}{ll}
x 1=\{a, b, c\}, \quad & X_{r}(x 1)=\left\{\{a, b, c\},\left\{a^{\prime}, c^{\prime}\right\},\left\{a, c^{\prime}\right\},\left\{a^{\prime}, c\right\}\right\} \\
& Y_{r}(x 1)=\left\{\{g, h\},\left\{g^{\prime}, i\right\},\left\{g, i^{\prime}\right\},\left\{g^{\prime}, h^{\prime}\right\}\right\} \\
& Y_{r}(x 1 z 1)=\left\{\{g, h\},\left\{g, i^{\prime}\right\}\right\}
\end{array}
$$

It is assumed that:

$$
\begin{array}{lll}
a \sim b \sim a^{\prime} & ; & c \sim c^{\prime} ; \\
g \sim g^{\prime} & ; & h \sim h^{\prime} ; i \sim i^{\prime} ; \\
z 1 \sim z 1^{\prime} & ; & z 2 \sim z 2^{\prime} .
\end{array}
$$

Therefore $\quad\left\{g^{\prime}, i\right\} \cong\left\{g, i^{\prime}\right\}$,

$$
\left\{g^{\prime}, h^{\prime}\right\} \cong\{g, h\}
$$

so $Y_{r}(x 1) \cong Y_{r}(x 1 z 1)$, and by similar reasoning we must have $Y_{r}(x 1) \cong Y_{r}(x 1 z 2)$.

We say fuzzy multivalued $X \approx \mapsto \longrightarrow Y$ is satisfied in $r 2$.

We now propose the set of fuzzy functional and multivalued dependencies inference rules over a set of atributes $U$. The first three for fuzzy functional dependencies are repeat here.

A1: Reflexivity for fuzzy functional dependencies (FFD)

If $Y \subseteq X$ then $X \approx Y$.

A2: Augmentation for FFD

If $X \approx Y$ holds, then $X Z \approx Y Z$ holds.

A3: Transitivity for FFD

If $X \approx Y$ and $Y \approx Z$ hold, then $X \approx Z$ holds. 
A4: Complementation for fuzzy multivalued dependencies (FMVD)

If $X \approx \longrightarrow Y$ holds, then $X \approx \longrightarrow$, where $Z=R-X Y$.

A5: Augmentation for FMVD

If $X \approx \longrightarrow Y$ holds, then $X Z \approx \longrightarrow Y Z$ holds.

A6: Transitivity for FMVD

If $X \approx \longrightarrow Y$ and $Y \approx \longrightarrow Z$ hold then $X \approx(Z-Y)$ holds.

Last two axioms that relate fuzzy functional and fuzzy multivalued dependencies are also similar to classical cases.

A7: If $X \approx Y$ holds, then $X \approx \rightarrow Y$.

A8: If $X \approx \longrightarrow Y$ holds, $Z \subseteq Y, W \cap Y=\emptyset$, and $W \approx Z$, then $X \approx Z$ holds.

Lemma 5.1. The set of axioms (A1-A8) are sound. That is, if the fuzzy dependency (FFD or FMVD) is deduced from a set of FFDs and FMVDs, $G$, using the axioms, then it is true in any relation in which the dependencies of $G$ are true.

Proof. By Lemma 4.1, the axioms A1-A3 is sound.

(A4) Complementation for fuzzy multivalued dependencies (FMVD)

If $X \approx ⿱ \longleftrightarrow Y$ holds, then $X \approx \longrightarrow, Z$, where $Z=R-X Y$.

We shall prove that, if for every $X Z$-value $x z$ that appears in $r$ we have $Y(x) \cong Y(x z)$ then $Z(x) \cong$ $Z(x y)$ for every $X Y$-value $x y$ that appears in $r$. Obviously, $Z(x y) \subseteq Z(x)$. Therefore, we only need to show

$$
\forall z_{0}\left(Z(x) \exists z^{\prime} \in Z(x y): z_{0} \approx z^{\prime} .\right.
$$

Let $t, t_{0} \in r$, where $t=\langle x, y, z\rangle, t_{0}=\left\langle x_{0}, y_{0}, z_{0}\right\rangle$. Since $z_{0} \in Z(x)$, we have $x_{0} \approx x$, which implies, $y \in Y\left(x_{0}\right)$. On the other hand $Y(x 0) \cong Y\left(x_{0} z_{0}\right)$, we have also $\exists t_{1}=\left\langle<x_{1}, y_{1}, z_{1}\right\rangle \in r$ such that $y_{1} \in Y\left(x_{0} z_{0}\right)$ and $y \approx y_{1}$. It means that $x_{0} \approx x_{1}, z_{0} \approx z_{1}$ and $y \approx y_{1}$. By transitivity of equivalence relation $(\approx)$, we get $x \approx x_{1}$. Consider tuple $t_{1}$, we found the existing of $z^{\prime}$ in $(*)$ is pointed (let $\left.t^{\prime}=t_{1}\right)$, i.e. $r$ satisfies $X \approx \longrightarrow Z$.

(A7) If $X \approx Y$ holds, then $X \approx \longrightarrow Y$.

We need to show

$$
Y(x) \cong Y(x z) \forall t=\langle x, y, y\rangle \in r .
$$

Let $y_{0} \in Y(x)$, clearly $x_{0} \approx x$. Because $X \approx Y$ is valid in $r$, we have $y_{0} \approx y$. It is easy to see that $y \in Y(x z)$ and $y_{0} \approx y$. The proof is complete.

(A8): If $X \approx \longrightarrow Y$ holds, $Z \subseteq Y, W \cap Y=\emptyset$, and $W \approx Z$, then $X \approx Z$ holds.

Assume the contrary that we have a fuzzy relation $r$ in which $X \approx Y$ and $W \approx Z$ hold, where $Z \subseteq Y, W \cap Y=\emptyset$ but $X \approx Z$ does not hold.

Thus, $\exists t_{1}, t_{2} \in r$ such that $\left(t_{1}[X] \approx t_{2}[X]\right)$ is true but $\left(t_{1}[Z] \approx t_{2}[Z]\right)$ is not valid. $(* * *)$

Obviously $t_{2}[Y] \in Y\left(t_{1}[X]\right)$, from $t_{1}[X] \approx t_{2}[X]$. Since $X \approx \longrightarrow Y$ holds then $\exists t_{3} \in r: t_{3}[Y] \in$ $Y\left(t_{1}[X] t_{1}[R-X Y]\right)$ and $t_{3}[Y] \approx t_{2}[Y]$, which implies

$$
\begin{aligned}
t_{3}[X] & \approx t_{1}[X], \\
t_{3}[R-X Y] & \approx t_{1}[R-X Y], \\
t_{3}[Y] & \approx t_{2}[Y] .
\end{aligned}
$$

From $W \cap Y=\emptyset$, combining with (1) and (2), we have

$$
t_{3}[W] \approx t_{1}[W] \text {. }
$$

From $Z \subseteq Y$ and $(3)$, we have also $t_{3}[Z] \approx t_{2}[Z]$.

Since our contrary assumption $(* * *)$ and transitivity of equivalence relation $(\approx)$, it can be seen that $\left(t_{3}[Z] \approx t_{1}[Z]\right)$ does not hold in $r(5)$.

But (4) and (5) contradicts $W \approx Z$ holds in $r$. The proof is complete. 
Proof of (A5) easy to show from definition of FMVD and properties of equivalence relation $(\approx)$. Techniques of proof for (A6) are similar to those used in [4].

We also suppose that procedure of proof for the completeness of above inference axioms is similar to the classical case.

\section{CONCLUSIONS}

We have suggested the structure for representing uncertain information in the form of relational database. The models, which are given by B. P. Buckles and F. E. Petry [2] and by A. K. Mazumdar $[1,6]$, are only special cases. Based on the concept of redundancy on a set of tuples, the definitions of fuzzy dependencies (fuzzy functional dependency and fuzzy multivalued dependency) are proposed. It is interesting to note that the set of inference rules, which is similar to classical case [7], is sound and complete as well.

In order to continue, we have already begun some studies: research for extending the relational algebra in this model, and extension of this model such that it allows the presence of null values too.

\section{REFERENCES}

[1] Bhattacharjee T. K, Mazumdar A.K., Axiomatisation of fuzzy multivalued dependencies in a fuzzy relational data model, Fuzzy Sets and Systems 96 (1998) 343-352.

[2] Buckles B.P and Petry E., A fuzzy representation of data for relational databases, Fuzzy Sets and Systems 7 (1980) 213-226.

[3] Codd E.F., A relational model of data for large shared data banks, Commun. ACM 13 (6) (1970) 377-387.

[4] Ho Thuan, Ho Cam Ha, Huynh Van Nam, Some comments about "Axiomatisation of fuzzy multivalued dependencies in a fuzzy relational data model", Journal of Computer Science and Cybernetics 16 (4) (2000) 30-33.

[5] Petry E. and Bosc P., Fuzzy Databases Principles and Applications, Kluwer Academic Publishers, 1996.

[6] Raju K. V. and Mazumdar A. K., Functional Dependencies and lossless join decomposition of fuzzy relational database system, ACM Trans, Database System 13 (1988) 129-1966.

[7] Ullman J. F., Principles of Database Systems, 2nd Ed, Computer Science Press, Rockvill, MD, 1984.

[8] Zadeh L. A., Fuzzy sets, Inform. Control 12 (1965) 338-353.

[9] Zadeh L. A., Fuzzy sets as a basis for a theory of possibility, Fuzzy Sets and Systems 1 (1978) $3-28$. 